\title{
Flapping dynamics of an inverted flag in a uniform flow
}

\author{
Jaeha Ryu, Sung Goon Park, Boyoung Kim, Hyung Jin Sung* \\ Department of Mechanical Engineering, KAIST, 291 Daehak-ro, Yuseong-gu, Daejeon 305-701, Republic of Korea
}

\begin{abstract}
The flapping motions of an inverted flag in a uniform flow were simulated using the immersed boundary method. The strain energy of the inverted flag was used as an indicator of the energy harvesting system efficiency. The flapping dynamics of and vortical structures around the inverted flag were examined in terms of the bending rigidity $(\gamma)$ and the Reynolds number (Re). Three flapping motion modes were observed: a deflected mode, a flapping mode, and a straight mode. A mode intermediate between the flapping mode and the straight mode was identified, the biased mode. The vortical structures in the wake were characterized by three modes: a vortex pair; a vortex pair with a single vortex, and two vortex pairs, during half of the flapping period. The maximum mean strain energy $\left(E_{S}\right)$ was obtained when the vortical structures behind the inverted flag formed a vortex pair during the flapping mode.
\end{abstract}

\section{Introduction}

Much research in recent years has focused on the flow dynamics of flexible structures in a uniform flow and particularly on the flow dynamics related to energy harvesting systems. An energy harvesting system comprising piezoelectric patches attached to the surface of a flexible structure can convert the energy stored in solid deformations into an electric current that powers a purely resistive output circuit. Systems that can be exploited for energy harvesting are ubiquitous in nature, for example, rivers and straits, which can be used as the basis for an eco-friendly approach to generating energy without contaminating the surroundings.

Allen and Smits (2001) and Taylor et al. (2001) devised energy harvesting systems using the flapping of a flexible structure with a piezoelectric material attached to the structure's surface. Several researchers have suggested the design of energy harvesting systems based on a flexible flag that undergoes self-sustained oscillations. Such systems may be useful in a variety of fields, including oceanography and atmospheric sciences (Dunnmon et al., 2011; Giacomello and Porfiri, 2011). Energy harvesting systems based on piezoelectric patches attached to flexible structures operate through two energy transfer processes. First, the kinetic energy of a uniform flow is converted into strain energy within the flexible structure via interactions between the uniform flow and the flexible structure. Second, the electrical energy is harvested by the piezoelectric materials attached to the flexible structures (Michelin and Doare, 2013). The mechanisms through which the kinetic energy in a surrounding fluid is converted into strain energy in a flexible structure have been investigated in an effort to improve the energy harvesting efficiency (Ottman et al., 2003). Large deformations in flexible structures benefit the energy harvesting processes. Instabilities induced by large deformations in the flexible structures can significantly improve the energy harvesting system operation. Michelin and Doare (2013) increased the flow velocity to destabilize the flexible structures. Allen and Smits

\footnotetext{
* Corresponding author. Tel.: +82 42350 3027; fax: +82 423505027

E-mail address: hjsung@kaist.ac.kr (H.J. Sung).
} 
(2001) and Taylor et al. (2001) positioned bluff bodies ahead of the flexible structures to increase the instabilities of the structures. Recently, Kim et al. (2013) suggested the use of an inverted flag, in which the leading edge was free to move and the trailing edge was clamped. The inverted flag improved the amount of strain energy that was converted into the flag deformations from the surrounding fluid. The fluttering motions of the inverted flag were classified as a deflected mode, a flapping mode, and a straight mode. Many researchers have focused on developing piezoelectric devices to improve the energy harvesting efficiency (Loreto and Francesc, 2005; Shashank, 2007; Akaydin et al., 2010; Akcabay and Young, 2012). Michelin and Doare (2013) investigated the electrical and mechanical properties of piezoelectric patches. Sodano et al. (2004) focused on optimizing electric circuits that convert the solid deformations into an electric current.

Vortex structures generated in a wake represent the hydrodynamic footprint of the fluid interactions with the immersed structures. The vortex structures were analyzed quantitatively by Gharib et al. (1998), who defined the vortex formation time as the ratio between the length and the diameter of the ejected fluid column. The formation time provided an indicator of the vortex ring growth from the trailing jet. Kim et al. (2013) re-defined the formation time based on the peak-to-peak amplitude ratio $(A / L)$, the velocity at the tip of the inverted flag and the fluid velocity, to investigate the vortex dynamics generated in the wake of the fluttering inverted flag.

The immersed boundary method was adopted to handle the interaction between a uniform flow and flexible structures. In the method, the structures were imaginarily decomposed into the massive part and the massless part. The massive part is the only part on which the elastic force was exerted. The massless part interacted with the local fluid velocity. Both parts were united strongly by a stiff spring with damping between the two parts. Through this mechanism, the fluid equations and the solid governing equations were independently calculated with the momentum forcing which was the force of the stiff spring with damping (Peskin, 2002; Zhu and Peskin, 2002; Kim and Peskin, 2007). The improved version of the immersed boundary was proposed to handle the mass of a flag, in which the momentum forcing was added to induce the fluid velocity equivalent to the velocity on the immersed boundary (Huang et al., 2007; Huang and Sung, 2009).

In this study, we simulated the dynamics of an inverted flag in a uniform flow using the immersed boundary method. We focused on the mechanism by which the kinetic energy of the surrounding fluid was converted into strain energy in the inverted flag. The main purpose of the present study was to investigate the flow dynamics of the inverted flag as a function of the Reynolds number $(\mathrm{Re})$ and the bending rigidity $(\gamma)$. The numerical results were compared with experimental data reported by Kim et al. (2013). We examined the flapping shapes, the vortical structures, and the flapping dynamics in relation to the formation time and strain energy conversion. The next section describes the formulation used to simulate an inverted flag in a uniform flow and the numerical method derivation. In Section 3, the numerical results and discussion are presented. Finally, a summary is provided in Section 4.

\section{Problem formulation}

A schematic diagram of the inverted flag and coordinate system is shown in Fig. 1. An inverted flag with a free leading edge and a clamped trailing edge was subjected to a uniform flow. The interactions between the inverted flag and the surrounding fluid were modeled using the immersed boundary method, in which the momentum forcing was added to the Navier-Stokes equations. The fluid was governed by the continuity equation and the incompressible Navier-Stokes equations,

$$
\begin{aligned}
& \rho_{0}\left(\frac{\partial \boldsymbol{u}}{\partial t}+\boldsymbol{u} \cdot \nabla \boldsymbol{u}\right)=-\nabla p+\mu \nabla^{2} u+f, \\
& \nabla \cdot \boldsymbol{u}=0
\end{aligned}
$$

where $\boldsymbol{u}$ is the velocity vector, $p$ is the pressure, $\rho_{0}$ is the flow density, $\mu$ is the dynamic viscosity, and $\boldsymbol{f}$ is the momentum forcing used to enforce the no-slip boundary conditions along the immersed boundary.

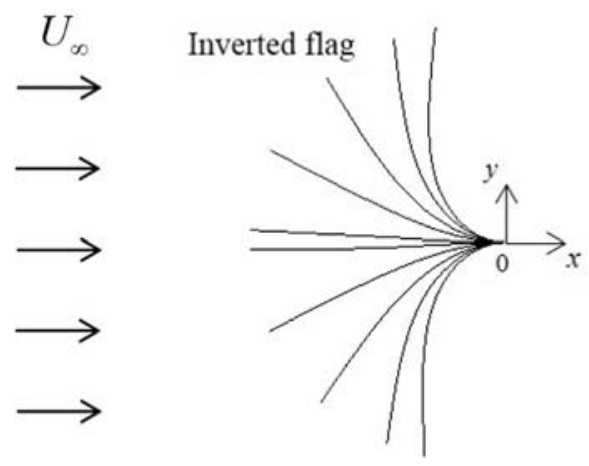

Fig. 1. Schematic diagram of the elastic inverted flag. 
The position of the inverted flag is denoted $\boldsymbol{X}(s)$ and is governed by the equation

$$
\rho_{1} \frac{\partial^{2} \boldsymbol{X}}{\partial t^{2}}=\frac{\partial}{\partial s}\left(T \frac{\partial \boldsymbol{X}}{\partial s}\right)-\frac{\partial^{2}}{\partial s^{2}}\left(\gamma \frac{\partial^{2} \boldsymbol{X}}{\partial s^{2}}\right)-\boldsymbol{F},
$$

where $s$ is the arc length, $\rho_{1}$ is the density difference between the fluid and the flag, $T$ is the tension force along the flag axis, $\gamma$ is the bending rigidity, and $\boldsymbol{F}$ is the Lagrangian forcing exerted on the flag by the flow. Eqs. (1)-(3) are non-dimensionalized using various characteristic scales. The flow density $\rho_{0}$ was used for normalizing the density, the flexible flag length $L$ for the length, the uniform velocity $U_{\infty}$ for the velocity, $L / U_{\infty}$ for the time, $\rho_{0} U_{\infty}^{2}$ for the pressure $p, \rho_{1} U_{\infty}^{2}$ for the tension force $T$, $\rho_{1} U_{\infty}^{2} / L$ for the momentum exerted on the fluid $f, \rho_{1} U_{\infty}^{2} / L$ for the Lagrangian momentum $\boldsymbol{F}$, and $\rho_{1} U_{\infty}^{2} L^{2}$ for the bending rigidity $\gamma$. The non-dimensionalized forms of Eqs. (1) and (3) are

$$
\begin{aligned}
& \frac{\partial \boldsymbol{u}}{\partial t}+\boldsymbol{u} \cdot \nabla \boldsymbol{u}=-\nabla p+\frac{1}{\operatorname{Re}} \nabla^{2} \boldsymbol{u}+\boldsymbol{f}, \\
& \frac{\partial^{2} \boldsymbol{X}}{\partial t^{2}}=\frac{\partial}{\partial s}\left(T \frac{\partial \boldsymbol{X}}{\partial s}\right)-\frac{\partial^{2}}{\partial s^{2}}\left(\gamma \frac{\partial^{2} \boldsymbol{X}}{\partial s^{2}}\right)-\boldsymbol{F},
\end{aligned}
$$

where Re indicates the Reynolds number normalized by $\rho_{0} U_{\infty} L / \mu$. The non-dimensional continuity equation took the form of Eq. (2). The inverted flag had a free leading edge and a clamped trailing edge. At the free leading edge $(s=L)$, we have

$$
T=0, \quad \frac{\partial^{2} \boldsymbol{X}}{\partial s^{2}}=(0,0), \quad \frac{\partial^{3} \boldsymbol{X}}{\partial s^{3}}=(0,0) .
$$

At the clamped trailing edge $(s=0)$,

$$
\boldsymbol{X}=\boldsymbol{X}_{0}, \quad \frac{\partial \boldsymbol{X}}{\partial s}=(-1,0) .
$$

As shown in Fig. $1, \boldsymbol{X}_{0}$ is $(0,0)$, the clamped edge of an inverted flag.

The momentum forcing term $\boldsymbol{F}$, which was added to the solid governing equation, was derived using the feedback law

$$
\boldsymbol{F}=\alpha \int_{0}^{t}\left(\boldsymbol{U}_{i b}-\boldsymbol{U}\right) d t^{\prime}+\beta\left(\boldsymbol{U}_{i b}-\boldsymbol{U}\right),
$$

where $\alpha$ and $\beta$ are large negative free constants $\left(\alpha=-1.74 \times 10^{7}, \beta=-2.60 \times 102\right)$ defined by Shin et al. (2008), $\boldsymbol{U}_{i b}$ is the fluid velocity on the immersed boundary obtained by interpolation, $\boldsymbol{U}$ is the velocity of an inverted flag expressed by $\boldsymbol{U}=d \boldsymbol{X} /$ $d \boldsymbol{t}$. In Eq. (8), the fluid on the immersed boundary and the inverted flag are connected by a virtual stiff spring with damping. The immersed boundary and the inverted flag moved together due to a stiff connection applied between the immersed boundary and the inverted flag. For this reason, the velocities of the fluid on the immersed boundary and the inverted flag were equal, leading to no-slip boundary conditions on the surface of the inverted flag.

The interaction force between the surrounding fluid and the inverted flag was obtained using the Dirac delta function. The velocity on the immersed boundary was interpolated according to

$$
\boldsymbol{U}_{i b}(s, t)=\int_{\Omega} \boldsymbol{u}(\boldsymbol{x}, t) \delta(\boldsymbol{X}(s, t)-\boldsymbol{x}) d \boldsymbol{x} .
$$

The Lagrangian forcing was distributed to the nearby grid points

$$
\boldsymbol{f}(\boldsymbol{x}, t)=\rho \int_{\Gamma} \boldsymbol{F}(s, t) \delta(\boldsymbol{x}-\boldsymbol{X}(s, t)) d s,
$$

where the density ratio $\rho=\rho 1 /\left(\rho_{0} L\right)$ comes from non-dimensionalization. We set $\rho=1$, indicating that the density of the inverted flag is two times larger than that of the fluid density $\left(\rho_{0}\right)$. Eqs. (8) and (10) show that the immersed boundary was forced to move along with the local fluid. In this study, the fluid equation was solved using the fractional step method (Kim et al., 2002), and the structure equation was solved based on a study performed by Huang et al. (2007). The numerical procedure is described in detail in Huang et al. (2007). Several trial calculations were repeated to monitor the sensitivity of the results to the domain size and the grid size. The domain size $8 L \times 8 L$ was chosen to show the vortical structures behind the inverted flag, where $L$ is the length of the inverted flag. Three grids were tested: $\left(N_{x} \times N_{y}\right)=(257 \times 161),(513 \times 225)$, and $(1025 \times 353)$. The outcome with the grid $(513 \times 225)$ was satisfactory in this simulation. The time step of the simulation was $10^{-5}$.

\section{Results and discussion}

\subsection{Groundwork for the simulation}

An inverted flag in a uniform flow is beneficial for energy harvesting systems due to the large instabilities of the flag (Kim et al., 2013). The flapping mode of an inverted flag provides highly efficient energy conversion due to the large peak-to-peak amplitude $(A / L) . A / L$ and the bending rigidity $(\gamma)$ are proportional to the mean strain energy. The bending rigidity is a 


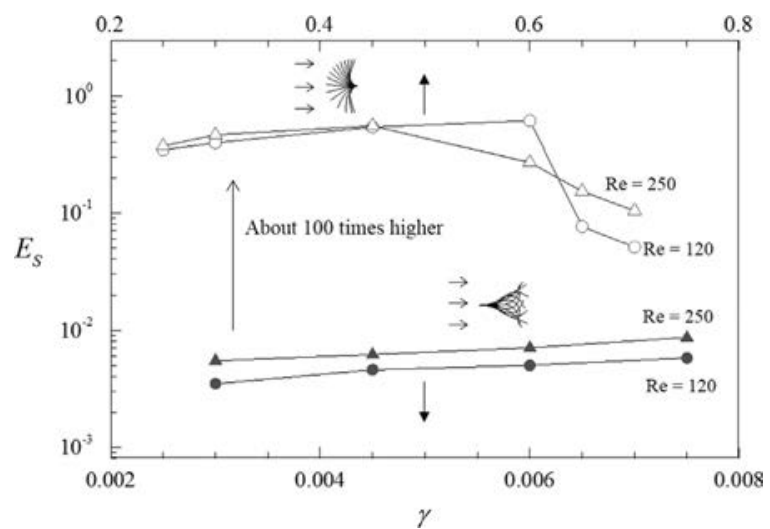

Fig. 2. Comparison of the mean strain energies in an inverted flag or in a typical flag.

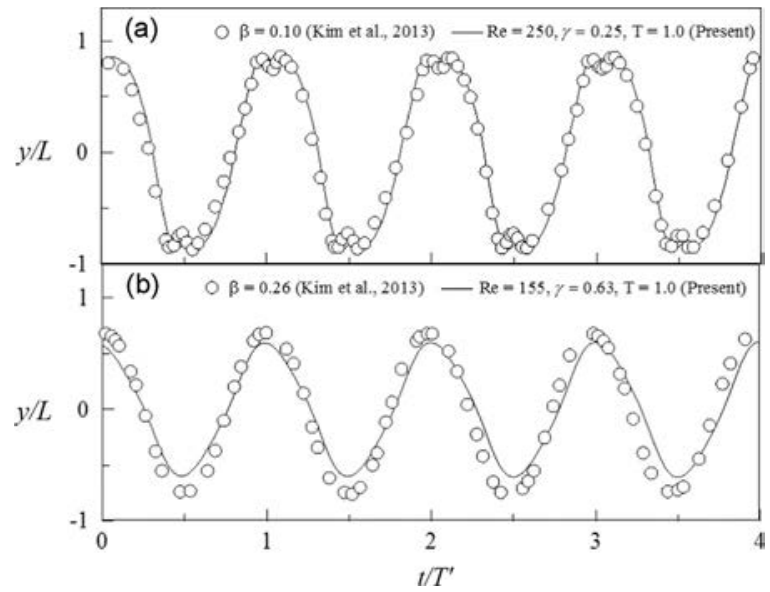

Fig. 3. Time history of the $y$-coordinate of the tip at (a) $\beta=0.10$ and $\operatorname{Re}=250, \gamma=0.25$; and (b) $\beta=0.26$ and $\operatorname{Re}=155, \gamma=0.63$.

constant value which decides the magnitude of bending momentum term of the non-dimensionalized flag momentum equation.

The differences in the mean strain energy between an inverted flag and a conventional typical flag were compared. Typical flags, with a pinned leading edge and a free trailing edge, have been examined in many previous studies for use in energy harvesting systems (Allen and Smits, 2001; Taylor et al., 2001; Michelin and Doare, 2013). Fig. 2 displays the differences between the mean strain energy in an inverted flag or in a typical flag under the same flow characteristics and elastic properties. The bending rigidity of the inverted flag was 100 times larger than that of the typical flag, since the typical flag with the given bending rigidity did not display self-sustained flapping. Here, 'self-sustained flapping' refers to a continuous flapping motion without disturbance. Researchers have attempted to induce self-sustained flapping motions by increasing the flow velocity or positioning bluff bodies ahead of a flag. As indicated in Fig. 2, the mean strain energy in the inverted flag was about 100 times higher than that in the typical flag. This means that the inverted flag was more efficient than the typical flag in converting the kinematic fluid energy into the flag strain energy within the energy harvesting system framework.

Our numerical results were compared with the experimental data reported by Kim et al. (2013). Fig. 3 indicates the time histories of the non-dimensional $y$-coordinates of the tip, revealing that the numerical results obtained in the present simulation agreed well with the experimental data. In the experiment, the bending stiffness $(\beta=0.10)$ was the lowest and $\beta=0.26$ was the highest to display the self-sustained flapping motion. The simulation for matching with the experiment $(\beta=0.10)$ was made at $\operatorname{Re}=250$ and $\gamma=0.25$. In the experiment, the flow velocity was $4.2 \mathrm{~m} / \mathrm{s}$ for $\beta=0.26$ and $6.7 \mathrm{~m} / \mathrm{s}$ for $\beta=0.10$, respectively. This velocity ratio was used for scaling $(\beta=0.26)$, i.e., $\operatorname{Re}=250 \times 0.63=155$ and $\gamma=0.25 / 0.63^{2}=0.63$.

\subsection{Flapping dynamics of an inverted flag}

Fig. 4 shows a phase diagram of the flag motions over the parameter space spanned by Re and the bending rigidity $(\gamma)$. For $\operatorname{Re}<50$, the flapping mode and the straight mode did not form because the viscous force was more dominant than the inertial force. In the range $100 \leq \operatorname{Re} \leq 250$, the deflected mode, the flapping mode, and the straight mode occurred in a regular sequence, depending on the bending rigidity of the flag. A new mode intermediate between the flapping mode and 


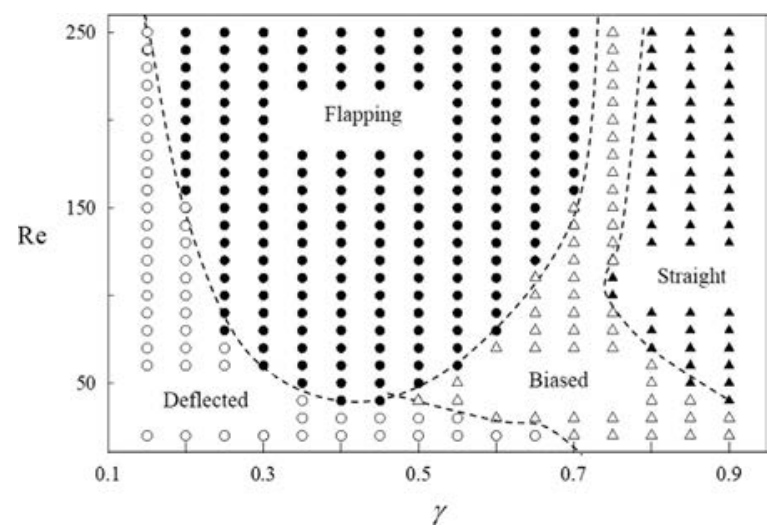

Fig. 4. The phase diagram over the parameter space spanned by Re and the bending rigidity $(\gamma)$.

the straight mode was observed and was called the biased mode. The geometric shape of the biased mode was similar to that of the straight mode, but the deflection of the leading edge $|\tilde{y}| / L$ of the biased mode was larger than that of the straight mode. Here, $|\tilde{y}|$ is the maximum deflection of the tip. The inverted flag flapped continuously or had a stationary position (after the first few time steps) based on different conditions. When the inverted flag had a stationary position, its deflection was the criterion for determining the straight, deflected or biased straight modes. The inverted flag in a deflected mode maintain a highly curved shape where the deflection of the leading edge is larger than 0.3 , i.e., $|\tilde{y}| / L \geq 0.3$. When the deflection of the inverted flag in a stationary position is less than 0.1 , i.e., $|\tilde{y}| / L \leq 0.1$, it is defined as the straight mode. When the deflection is within the range of $0.1<|\tilde{y}| / L<0.3$, it is defined as the biased mode. The mean strain energy in the biased mode was about $10-20 \%$ of the maximum mean strain energy, whereas the mean strain energy in the straight mode was almost zero. The flapping mode was characterized by an up and down fluttering motion through the center $y=0$.

The peak-to-peak amplitude $(A / L)$ is one of the most important characteristics of an inverted flag flapping. A high value of $A / L$ causes high curvatures and it leads that high mean strain energies may be obtained. Fig. 5 plots the variations in $A / L$ and the Strouhal number (St) as a function of the bending rigidity. $A / L$ was found to be insensitive to Re. St increased slightly as Re increased. $A / L$ increased significantly as the dynamics shifted from the deflected mode to the flapping mode, and decreased gradually as the dynamics shifted from the flapping mode to the biased mode. The inverted flag in the biased mode or the straight mode did not flap, and $A / L$ was almost equal to zero. The inverted flag in the flapping mode was characterized by $A / L \geq 1.6$, in agreement with the results reported by Kim et al. (2013). In this study, the flapping mode was divided into two distinct regions, depending on $A / L$ : a high-amplitude flapping mode $(A / L \geq 1.6)$ and a low-amplitude flapping mode $(0.6 \leq A / L \leq 1.4)$. The highamplitude flapping mode was the most important phase of the dynamics because a high $A / L$ can lead to a high strain energy. Over the range $100 \leq \operatorname{Re} \leq 230$ the bending rigidity fell within the range $0.6<\gamma<0.65$. St increased gradually for an inverted flag in a high-amplitude flapping mode and decreased to a greater extent for an inverted flag in a low-amplitude flapping mode.

Fig. 6 superimposes the positions of inverted flags collected from 0 to 48 non-dimensional time with the interval of 0.24 . The black solid line indicates the stationary position and the black dashed line indicates the initial position. For $0.2<\gamma<0.45$, the superimposed inverted flags nearly coincided, with high peak-to-peak amplitudes $(A / L \geq 1.6)$. The observed plateau in $A / L$ corresponded to the morphological limit of the inverted flag, such as the clamped trailing edge. For $0.6<\gamma<0.7$, the peak-to-peak amplitudes of the superimposed inverted flags were relatively low. For $\gamma>0.75$, the superimposed flags did not deviate significantly from the stationary position. The low-amplitude flapping mode $(\gamma=0.6$ and $\gamma=0.7)$ and the biased mode $(\gamma=0.75)$ were identified schematically. The inverted flag in the biased mode flapped initially and then quickly stabilized (the black solid line). When the flag reached the stationary position, its deflection was within the range 0.1-0.3. The inverted flag displayed low-amplitude flapping mode motions for a bending rigidity of 0.7 , whereas the biased mode was preferred for a bending rigidity of 0.75 . The $y$ value of the tip in the stationary position was about 0.8 for a bending rigidity of 0.15 and about 0.2 for a bending rigidity of 0.75 .

\subsection{The efficiencies of the energy harvesting systems}

Fig. 7 shows the elastic mean strain energy $\left(E_{S}\right)$ and the mean drag coefficient $\left(C_{D}\right)$ under various Reynolds number conditions. $E_{S}$ is defined as $E_{S}=\frac{1}{T^{\prime}} \int_{0}^{T \prime} \frac{1}{2} \gamma \int_{0}^{L}\left(\frac{\partial^{2} X}{\partial S^{2}}\right)^{2} d s d t$, where $T^{\prime}$ is the flapping period and $s$ is the Lagrangian coordinate of the inverted flag. The strain energy could be maximized by increasing the area below the curve of $E_{S}$, thereby increasing the drag force. For this reason, the mean strain energy and the mean drag coefficient were correlated with the bending rigidity. For $\gamma=0.15$, the inverted flag remained in the deflected mode. As shown in Fig. 7(a), the mean strain energy and the mean drag coefficient decreased gradually in the deflected mode, whereas these parameters increased in the flapping mode. The bending rigidity that minimized the mean strain energy was 0.2 , and this value indicated the mode changes from a deflected mode to a flapping mode. A critical point was observed when $E_{S}$ and $C_{D}$ reached local minima. This point corresponded to the transition between the deflected mode and the flapping mode. The values of $E_{S}$ and $C_{D}$ in the high-amplitude flapping 
(a)

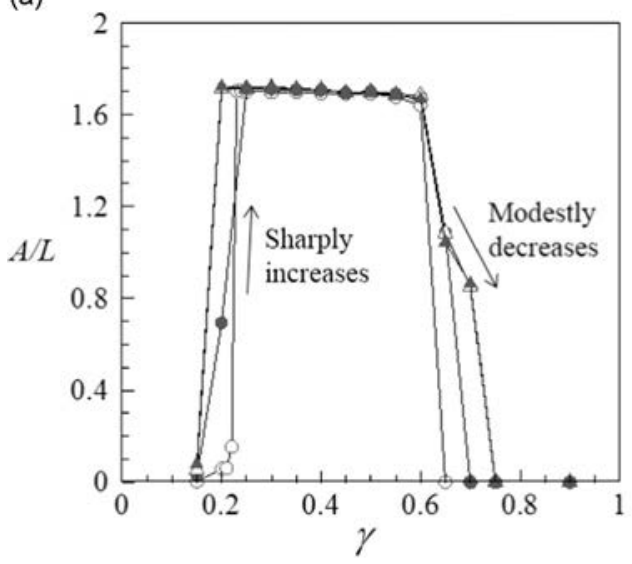

(b)

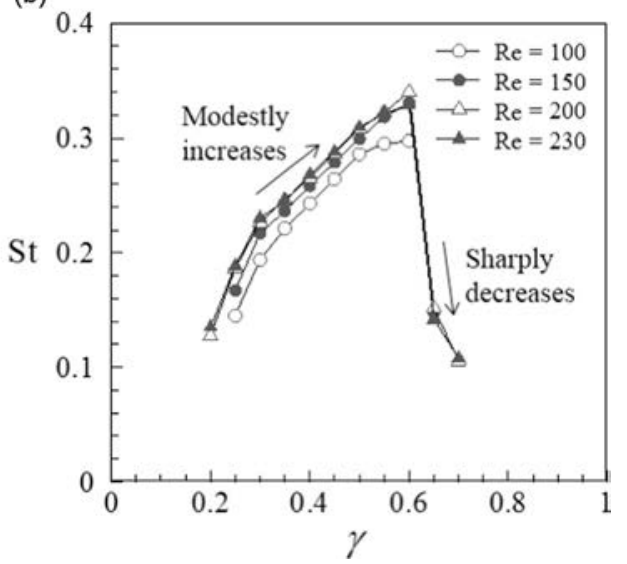

Fig. 5. (a) Peak-to-peak amplitude $A / L$ and (b) Strouhal number as a function of $\gamma$.
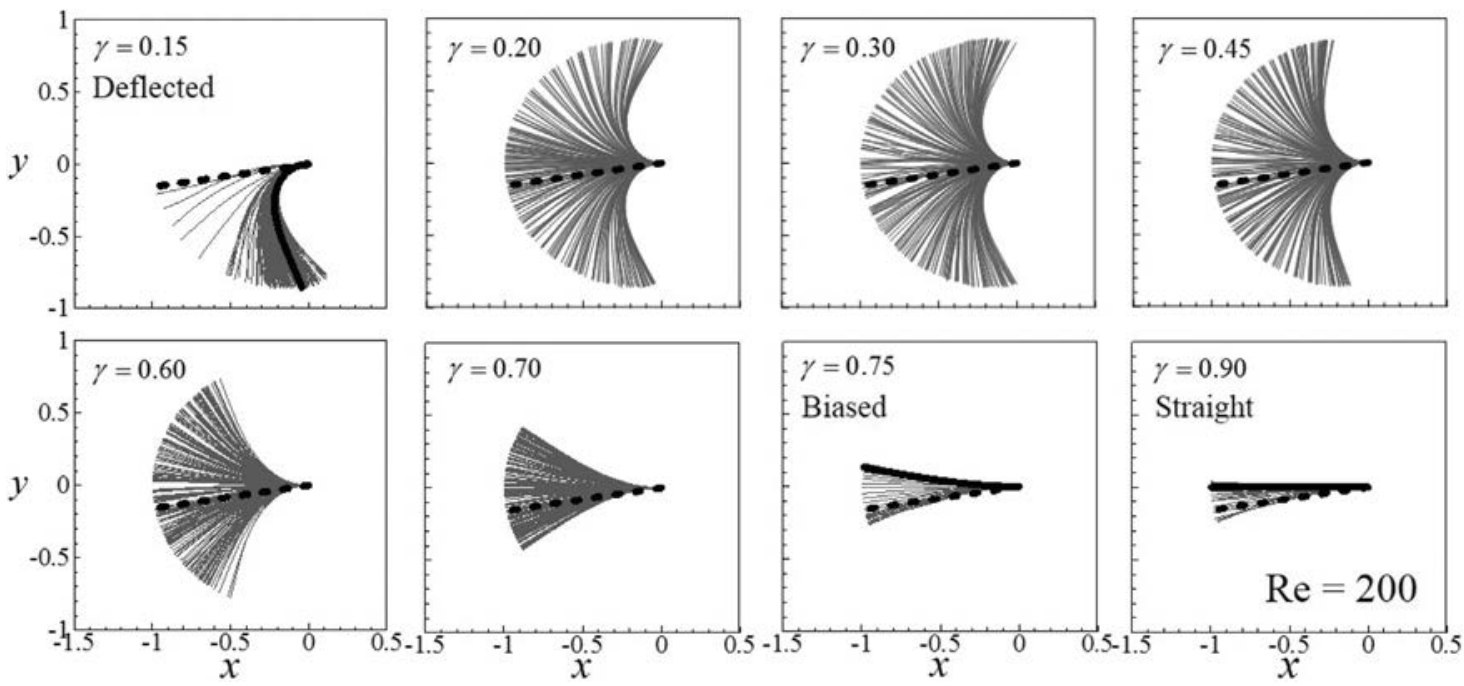

Fig. 6. Superimposed inverted flag positions for different values of $\gamma=0.15-0.90$. The red line indicates the stationary position of the inverted flag and the green line indicates the initial position. In a flapping mode, the red line was not indicated since the inverted flag flapped continuously and did not reach in a stationary state.

(a)

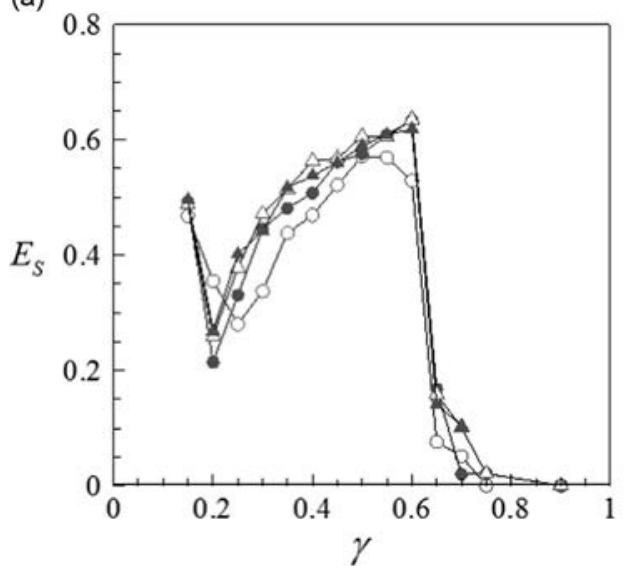

(b)

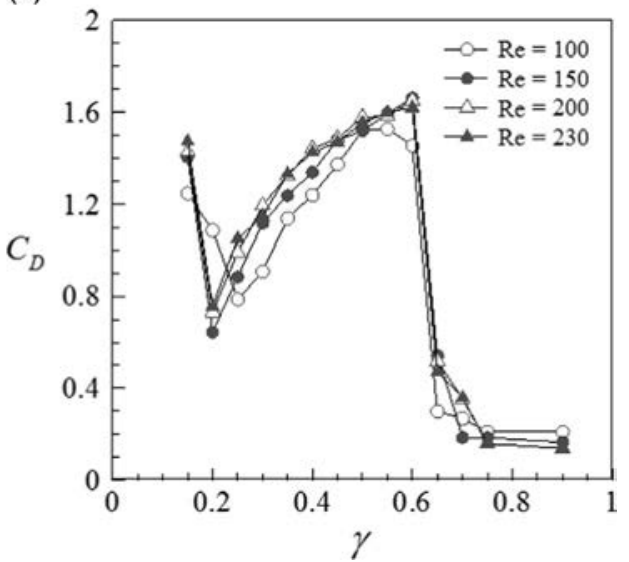

Fig. 7. (a) Elastic mean strain energy $E_{S}$, and (b) mean drag coefficient $C_{D}$, for various bending rigidities. 


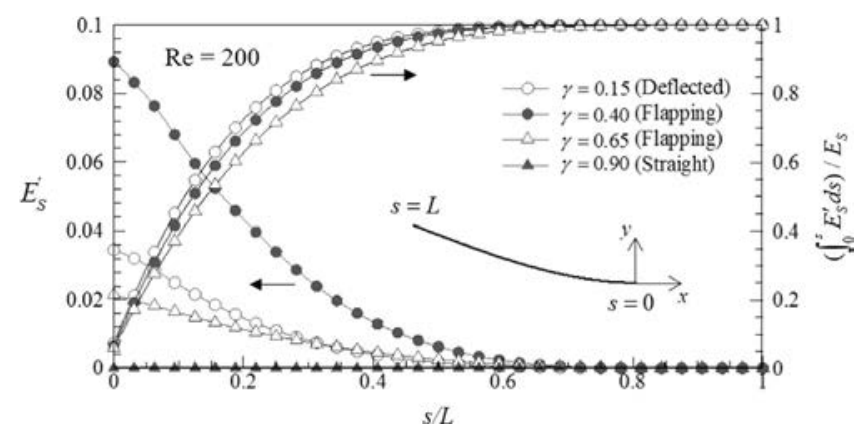

Fig. 8. Local strain energy $E_{S}^{\prime}$ and a cumulative sum of the local strain energy along the Lagrangian coordinate of the inverted flag.

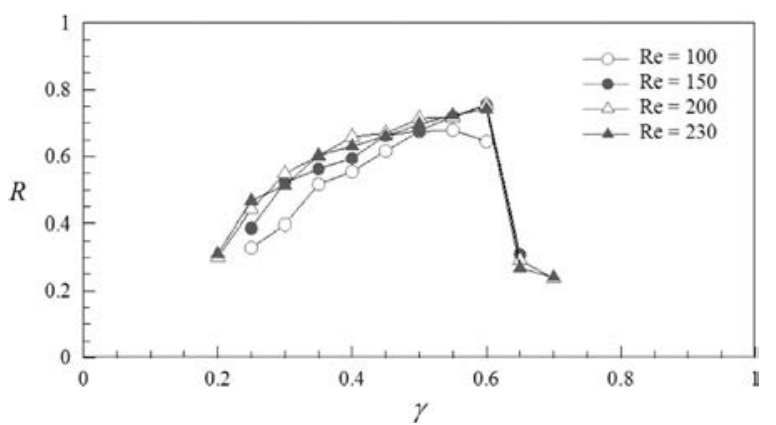

Fig. 9. The conversion ratio during the bending phase of the flapping mode.

mode increased as the bending rigidity increased and decreased rapidly as the mode transitioned from the high-amplitude flapping mode to the low-amplitude flapping mode. After the rapid drop in $E_{S}$ and $C_{D}$, the mode of the inverted flag transitioned to the biased mode. The values of $E_{S}$ and $C_{D}$ reached a maximum in the high-flapping amplitude mode. An inverted flag with a high bending rigidity moving in the high-amplitude flapping mode provided the greatest energy harvesting efficiency. The mean strain energy and the mean drag coefficient in the straight mode $(\gamma \approx 0.75-0.9)$ were very low because the deflections of these flags were almost zero. In general, $E_{S}$ and $C_{D}$ were insensitive to Re.

Fig. 8 shows the local strain energy $\left(E_{S}^{\prime}\right)$ defined as, $E_{S}^{\prime}=\frac{1}{T^{\prime}} \int_{0}^{T \prime} \frac{1}{2} \gamma\left(\frac{\partial^{2} \boldsymbol{X}}{\partial s^{2}}\right)^{2} d t$, and a cumulative sum of the local strain energy $\left(\int_{0}^{s} E_{S}^{\prime} d s\right)$ along the Lagrangian coordinate of the inverted flag. The local strain energy was maximized at the clamped trailing edge of the inverted flag. In all of the flapping modes, the local strain energy approached zero after $s / L=0.7$. The half of the inverted flag at the trailing edge $(s / L \approx 0-0.5)$ displayed a greater mean strain energy due to the trailing edge of the inverted flag underwent the greatest deflections. The local strain energy at $s / L=0.2$ was larger than the half of the maximum local strain energy. The inverted flag accumulated about $90 \%$ of the mean strain energy near the clamped edge $(s / L \approx 0-0.4)$. Fig. 8 indicates that the piezoelectric patches should be attached as close as possible to the trailing edge, where the local strain energy reaches a maximum value.

Kim et al. (2013) suggested using a conversion ratio $R=E_{S} / E_{K}$, defined as the ratio between the mean strain energy of an inverted flag $\left(E_{S}\right)$ and the mean kinetic energy of the uniform flow $\left(E_{K}=1 / 2 \rho_{1} U_{\infty}^{3}|\tilde{y}|\right)$ to compare the efficiencies of energy harvesting systems. Fig. 9 shows the conversion ratio plotted as a function of the bending rigidity. The conversion ratio range was smaller than the bending rigidity range, unlike the ranges of $E_{S}$ and $C_{D}$ plotted in Fig. 7, since the conversion ratio was only obtained in the flapping mode. As with $E_{S}$ and $C_{D}$, the conversion ratio reached a maximum in an inverted flag with a maximal bending rigidity undergoing high-amplitude flapping motions. The kinetic energy of a uniform flow with a given Re differed from the absolute value of $|\tilde{y}|$. The deflections measured during the high-amplitude flapping motions were nearly identical in all cases, and the kinetic energies were also equivalent. The conversion ratio followed a trend similar to that displayed by the mean strain energy, as shown in Figs. 7 and 9. These results suggested that the energy harvesting system efficiency could be estimated by measuring the strain energy of the inverted flapping flag. As depicted in Fig. 9, in the high-amplitude flapping mode, the maximum bending rigidity optimized the efficiency of the energy harvesting system. The maximum $R$ which can be the maximum efficiency of the system converged to about 0.77 . The bending rigidity for the maximum $R$ was almost the same as that of the maximum strain energy.

The formation time of the inverted flag, $F=\int_{t_{i}}^{t_{f}}\left\{\left[U+u_{x}(t)\right] /|\tilde{y}|\right\} d t$ discussed by Kim et al. (2013) provides a quantitative value that may be used to analyze the vortical structures present in the wake. Here, $u_{x}(t)$ is the velocity of the tip, $t_{f}$ is the time at which the inverted flag reaches its maximum deformation, $t_{i}$ is the time at which the inverted flag crosses the $y=0$ line, and $|\tilde{y}|$ is the maximum deflection of the tip at $t=t_{f}$. Previous studies examined formation times of $4.0 \leq F \leq 6.0$ in an inverted flag in the flapping mode. Kim et al. (2013) asserted that the formation number could be used to characterize the relationship between the optimal vortex formation and the efficient storage of strain energy. Fig. 10 shows the formation time as a function 
(a)



(b)



Fig. 10. (a) The formation number of the inverted flag. The formation numbers obtained from positive and negative values of $y$ were averaged. (b) The strain energy with respect to the formation number, for $\mathrm{Re}=100,150,200$, and 230.

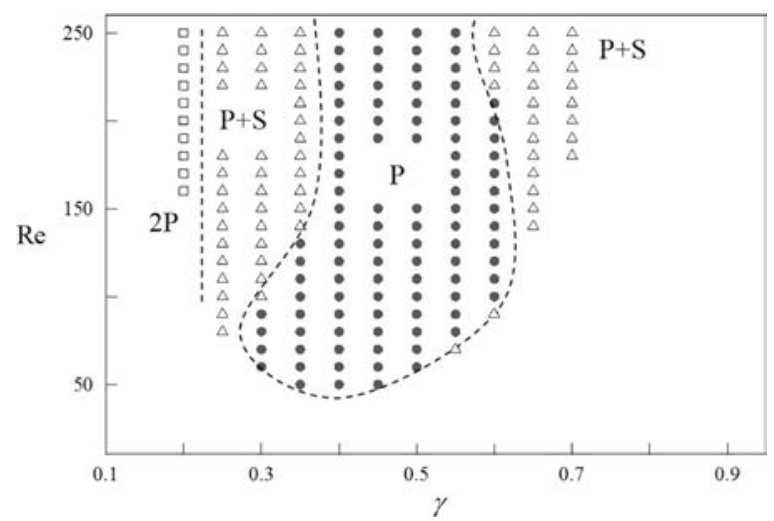

Fig. 11. Dynamical phase diagram of the vortical structure regimes formed behind an inverted flag, over the parameter space spanned by Re and $\gamma$.

of the bending rigidity and the relationship between the mean strain energy and the formation time. The vortex formation time was obtained over bending rigidity ranges in which the inverted flag was in the flapping mode, i.e., within $0.2<\gamma<0.7$. The formation time reached a minimum as the mean strain energy reached a maximum. The formation time obtained in the experiment (Kim et al., 2013) was higher than that of the present simulation since the experiment was performed at a higher Re number. The formation time in the present study was less than 4, implying that the vortex strength was not maximized.

As shown in Fig. 10(b), the formation time was inversely proportional to the mean strain energy. The asymptotic curve of the mean strain energy as a function of the formation time followed the relation $E_{S}=1.107-0.261 F$. The negative multiplier of the formation time indicated that the mean strain energy was inversely related to the formation time. The physical explanation for the inverse relationship may be obtained by examining the formation time equation. The formation time did not affect the free stream velocity because the free stream velocity did not change much in the inverted flapping system. The formation time changed most significantly during one quarter of the flapping period $\left(t_{f}-t_{i}\right)$ bounded by the point $t=t_{f}$ corresponding to the maximum deflection $|\tilde{y}|$ of the tip. The inverted flag had a relatively low bending rigidity in the high-amplitude flapping mode, and the low-amplitude flapping mode displayed a large vortex formation time (Fig. 10(a)). An inverted flag with a relatively low bending rigidity moving with high-amplitude flapping motions displayed a longer flapping period quarter $\left(t_{f}-t_{i}\right)$ compared to the corresponding values in other cases, due to the longer formation time. An inverted flag undergoing low-amplitude flapping motions displayed a smaller maximum deflection $|\tilde{y}|$ compared to the corresponding values in other cases, thereby increasing the formation time. For these reasons, the vortex formation time was inversely proportional to the mean strain energy, as shown in Figs. 7(a) and 10(a). Fig. 10(a) indicates that the vortical structures of the inverted flag with a high mean strain energy did not fully develop. The formation times reached values of 3.5-4.5 (Gharib et al., 1998) once the vortical structures in the wake had fully developed. The formation time of the inverted flag was around 2.0, once the mean strain energy had reached its maximum.

\subsection{Vortical structure formation}

The vortical structures produced by an inverted flag in a fluid flow depend on the bending rigidity and the Reynolds number, among other parameters. Williamson and Roshko (1988) classified the vortex shedding patterns behind an 
(a)

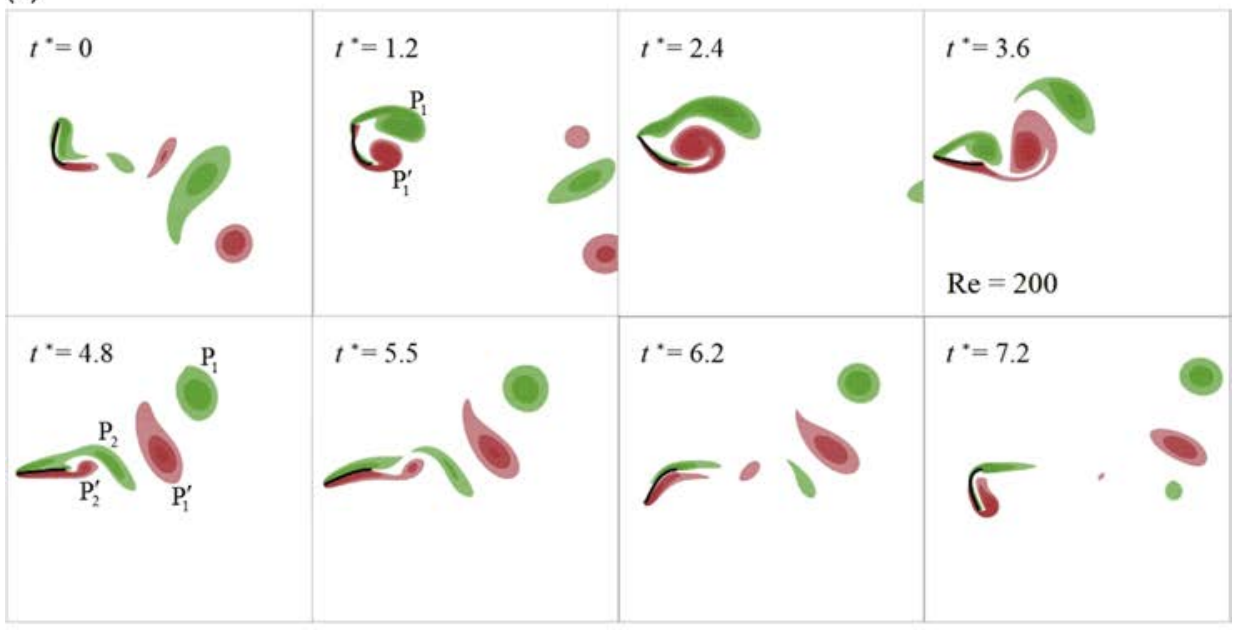

(b)

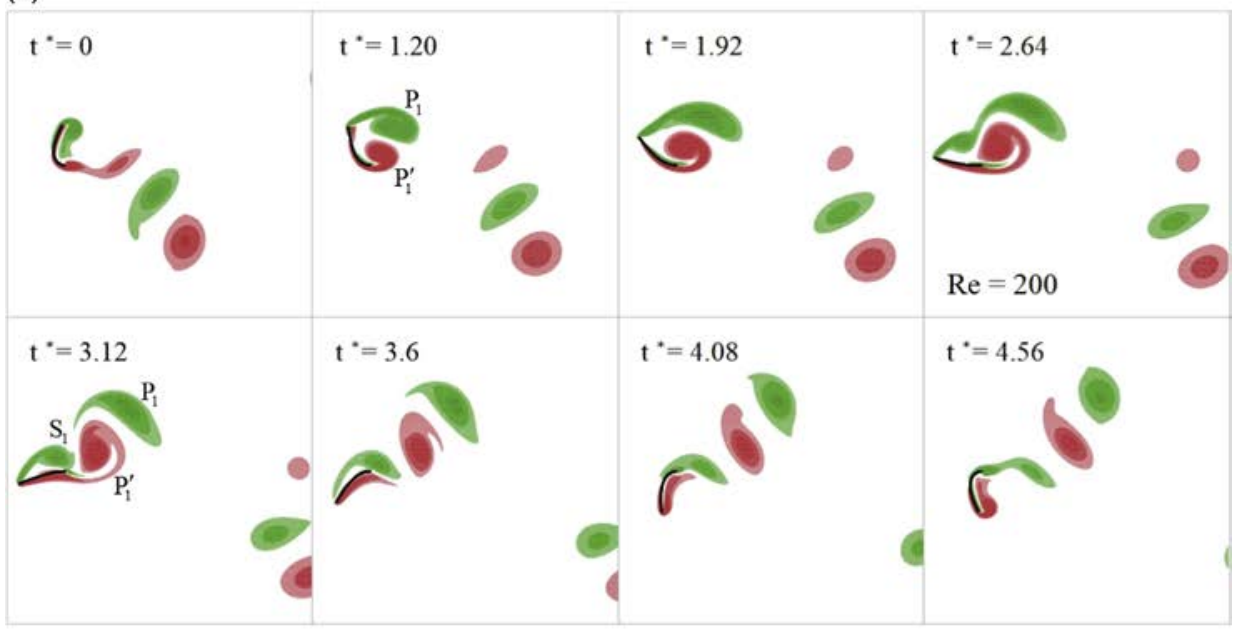

(c)

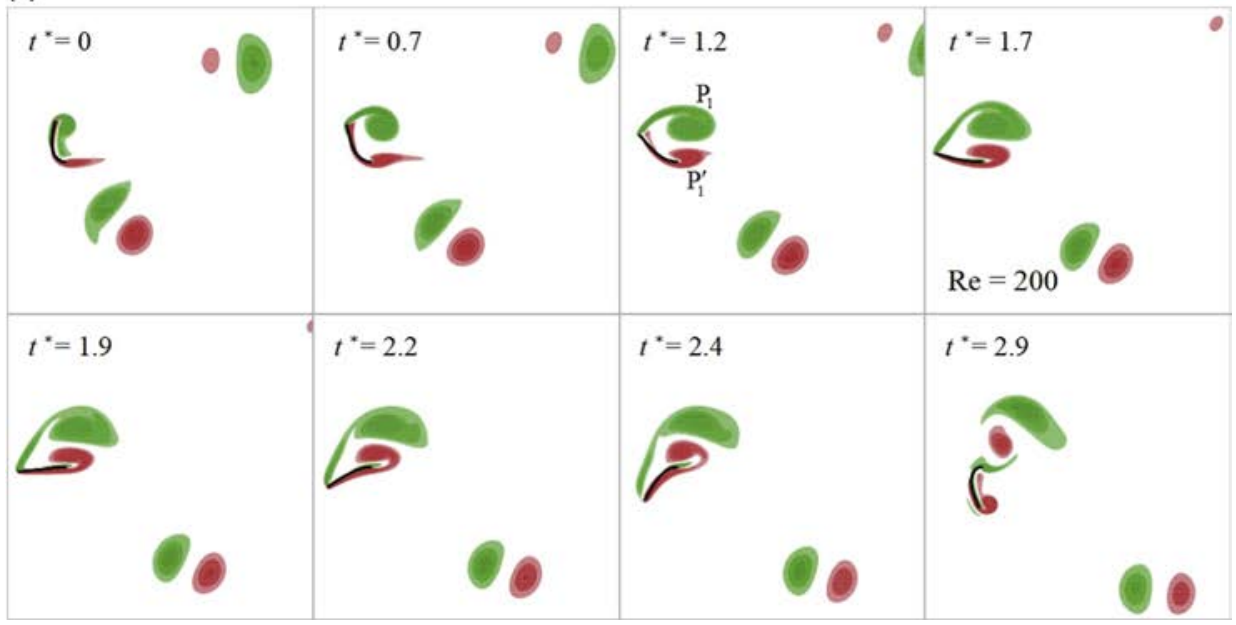

Fig. 12. The vortex formation process at (a) $\gamma=0.2$ (2P mode); (b) $\gamma=0.25(\mathrm{P}+\mathrm{S}$ mode); and (c) $\gamma=0.4$ (P mode).

oscillating cylinder. In their study, diagrams of the patterns were designated using combinations of $\mathrm{P}$, which indicated a vortex pair, $2 \mathrm{P}$, which indicated two vortex pairs, and $\mathrm{P}+\mathrm{S}$, which indicated a vortex pair with a single vortex. They considered the vortical structures formed during one oscillation period, and we adapted this idea in an analysis of an 


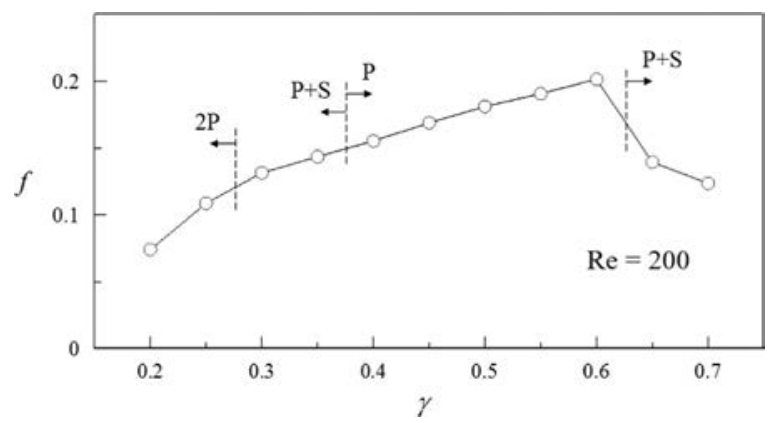

Fig. 13. Flapping frequency as a function of $\gamma$, annotated to indicate the vortical structure growth mode (2P mode, $\mathrm{P}+\mathrm{S}$ mode, and $\mathrm{P}$ mode).

inverted flag over one half-period of the flapping motion. The vortical structures formed by an inverted flag during one halfperiod were classified as displaying the $\mathrm{P}$ mode, $2 \mathrm{P}$ mode, and $\mathrm{P}+\mathrm{S}$ mode. Fig. 11 plots the vortical structure modes produced by an inverted flag over a bending rigidity range that supported flapping behavior. The vortical structures were influenced mainly by the bending rigidity. As the bending rigidity increased, the $2 \mathrm{P}$ mode, $\mathrm{P}+\mathrm{S}$ mode, $\mathrm{P}$ mode, and $\mathrm{P}+\mathrm{S}$ modes were observed.

Fig. 12 illustrates the formation process of the $2 \mathrm{P}$ mode, $\mathrm{P}+\mathrm{S}$ mode, and $\mathrm{P}$ mode during one-half of a flapping period. The vorticity contour was identified when the absolute vorticity was higher than $5 \%$ of the maximum vortex strength. As shown in Fig. 12(a), the 2P mode was initiated by detachment of a pair of vortical structures from both the leading and trailing edges as the inverted flag was deflected to one side $\left(t^{*}=0\right)$. The second pair of vortical structures detached from both edges as the inverted flag passed through the center $y=0\left(t^{*}=4.8\right)$. A total of 4 significant vortical structures were generated and detached during one-half of the flapping period. Fig. 12(b) shows that in the P+S mode, a pair of vortical structures was generated and detached from both the leading and trailing edges as the inverted flag was deflected to one side $\left(t^{*}=0\right)$. After the vortex pair had detached from the flag, a single vortical structure formed at the free leading edge $\left(t^{*}=2.6\right)$ and disengaged as the inverted flag deflected maximum to other side $\left(t^{*}=4.1\right)$. As visualized in Fig. 12(c), the P mode underwent a formation procedure similar to that observed in the $\mathrm{P}+\mathrm{S}$ mode, i.e., a pair of vortical structures was generated from the leading and trailing edges and the vortex provoked at the leading edge was separated as the inverted flag passed through the center $\left(t^{*}=1.7\right)$. A pair of the vortical structures was detached when the inverted flag was deflected to the opposite side $\left(t^{*}=2.4\right)$. However, no other vortical structures developed from the leading and trailing edges, unlike the $\mathrm{P}+\mathrm{S}$ mode. The three modes could be distinguished by the vortex formation behavior during the inverted flag passage through the position $y=0$ : as the inverted flag crossed the center line, the vortical structures were in the $2 \mathrm{P}$ mode if a pair of vortical structures detached; the vortical structures were in the $\mathrm{P}+\mathrm{S}$ mode if a single vortex detached; and the vortical structures were in the P mode if no vortical structures detached.

The vortical structure mode was affected by the flapping frequency of the inverted flag. Fig. 13 plots the flapping frequencies over a range of bending rigidities for $\mathrm{Re}=200$. The vortical structures could not be classified clearly according to the flapping frequency, but a trend was observed. Relatively high flapping frequencies ( $f$ of around 0.2 ) produced vortical structures in the $\mathrm{P}$ mode. Since the inverted flag flapping sequence was characterized by a high frequency, a pair of vortical structures developed. In the same way, the vortical structures formed in the $\mathrm{P}+\mathrm{S}$ mode for a flapping frequency of around 0.1 , and the vortical structures formed in the $2 \mathrm{P}$ mode for a flapping frequency of 0.07 . The flapping frequency provided a criterion by which the vortical structure modes could be distinguished. Low flapping frequencies provided the vortical structures with sufficient time to grow, thereby generating two pairs of vortical structures in a single flapping cycle. As the flapping frequency increased, the time permitted for vortical structure growth was reduced, thereby decreasing the number of vortical structures that formed.

\section{Conclusions}

We simulated the flow dynamics of an inverted flag with a free leading edge and a clamped trailing edge in a uniform flow. The simulation was conducted using the immersed boundary method, in which the solid motion equation and the fluid motion equation were solved separately by adding an interpolated momentum forcing term to each equation. The simulation results agreed well with the experimental data reported by Kim et al. (2013). The inverted flag has been suggested as a possible eco-friendly energy harvesting system. An inverted flag system appears to perform better than a typical flag system. We found that the peak-to-peak amplitude $(A / L)$ was not affected by Re and reached a plateau in the flapping mode as a result of the clamped conditions. $A / L$ influenced both the dynamics of the inverted flag and its energy harvesting efficiency. The mean strain energy and the conversion ratio were maximized for an inverted flag moving in the high-amplitude flapping mode. To maximize energy harvesting, piezoelectric patches may be attached as close as possible to the trailing edge of an inverted flag, where the strain energy is maximized. The elastic mean strain energy approached zero beyond $s / L=0.7$. The biased mode, which occurred between the flapping mode and the straight mode, was identified for the first time. The geometric shape of the inverted flag in the biased mode was similar to that in the straight mode, but the deflections in the biased mode were larger than those in the straight mode. The mean strain energy was inversely proportional to the formation number, and vortical structures did not fully develop during the inverted flag flapping sequence. The $\mathrm{P}, \mathrm{P}+\mathrm{S}$, and $2 \mathrm{P}$ vortex structure 
formation modes were observed during the flapping sequence, and the maximum mean strain energy was obtained from the $\mathrm{P}$ mode. The vortical structure classification scheme was closely related to the flapping frequency. A relatively high flapping frequency ( $f$ around 0.2 ) produced vortical structures in the P mode.

\section{Acknowledgments}

This work was supported by the Creative Research Initiatives (No. 2015-001828) program of the National Research Foundation of Korea (MSIP).

\section{References}

Akaydin, H.D., Elvin, N., Andreopoulos, Y., 2010. Wake of a cylinder: a paradigm for energy harvesting with piezoelectric materials. Experiments in Fluids 49, 291-304.

Akcabay, D.T., Young, Y.L., 2012. Hydroelastic response and energy harvesting potential of flexible piezoelectric beams in viscous flow. Physics of Fluids 24 , 054106.

Allen, J.J., Smits, A.J., 2001. Energy harvesting eel. Journal of Fluids and Structures 15, 629-640.

Dunnmon, J.A., Stanton, S.C., Mann, B.P., Dowell, E.H., 2011. Power extraction from aeroelastic limit cycle oscillations. Journal of Fluids and Structures 27 , $1182-1198$.

Gharib, M., Rambod, E., Shariff, K., 1998. A universal time scale for vortex ring formation. Journal of Fluid Mechanics 360, 121-140.

Giacomello, A., Porfiri, M., 2011. Underwater energy harvesting from a heavy flag hosting ionic polymer metal composites. Journal of Applied Physics 109, 084903.

Huang, W.-X., Shin, S.J., Sung, H.J., 2007. Simulation of flexible filaments in a uniform flow by the immersed boundary method. Journal of Computational Physics 226, 2206-2228.

Huang, W.-X., Sung, H.J., 2009. An immersed boundary method for fluid-flexible structure interaction. Computer Methods in Applied Mechanics and Engineering 198, 2650-2661.

Kim, K., Baek, S.-J., Sung, H.J., 2002. An implicit velocity decoupling procedure for incompressible Navier-Stokes equations. International Journal for Numerical Methods in Fluids 38, 125-138.

Kim, D., Cosse, J., Cerdeira, C.H., Gharib, M., 2013. Flapping dynamics of an inverted flag. Journal of Fluid Mechanics 736, R1.

Kim, Y., Peskin, C.S., 2007. Penalty immersed boundary method for an elastic boundary with mass. Physics of Fluids 19, 053103.

Loreto, M., Francesc, M., 2005. Optimum piezoelectric bending beam structures for energy harvesting using shoe inserts. Journal of Intelligent Material Systems and Structures 16, 835-845.

Michelin, S., Doare, O., 2013. Energy harvesting efficiency of piezoelectric flags. Journal of Fluid Mechanics 714, 489-504.

Ottman, G.K., Hofmann, H.F., Lesieutre, G.A., 2003. Optimize piezoelectric energy harvesting circuit using step-down converter in discontinuous conduction mode. IEEE Transactions on Power Electronics 18, 696-703.

Peskin, C.S. 2002. The immersed boundary method. Acta Numerica 11, 479-517.

Shashank, P., 2007. Advances in energy harvesting using low profile piezoelectric transducers. Journal of Electroceramics 19, 165-182.

Shin, S.J., Huang, W.-X., Sung, H.J., 2008. Assessment of regularized delta functions and feedback forcing schemes for an immersed boundary method. International Journal for Numerical Methods in Fluids 58, 263-286.

Sodano, H.A., Park, G., Inman, D.J., 2004. Estimation of electric charge output for piezoelectric energy harvesting. Strain 40, 49-58.

Taylor, G.W., Burns, J.R., Kammann, S.M., Powers, W.B., Welsh, T.R., 2001. The energy harvesting eel: a small subsurface ocean/river power generator. IEEE Journal of Oceanic Engineering 26, 539-547.

Williamson, C.H.K., Roshko, A., 1988. Vortex formation in the wake of an oscillating cylinder. Journal of Fluids and Structures 2, 355-381.

Zhu, L., Peskin, C.S., 2002. Simulation of a flapping flexible filament in a flowing soap film by the immersed boundary method. Journal of Computational Physics 179, 452-468. 